\section{Internacionalización Universitaria y Redes de Cooperación Sur-Sur}

Resumen: A lo largo del artículo se analizan las principales problemáticas que presenta la internacionalización universitaria en América Latina, cuando es incorporada en forma acrítica, y las potencialidades de disputar su sentido a partir de la promoción de políticas de cooperación sur-sur. Desde una perspectiva de análisis situada, relacional y política, se discute el concepto hegemónico de internacionalización, que se reproduce a partir de dinámicas de cooperación asimétricas norte-sur y en base a agendas de investigación exógenas a la Región Latinoamericana. Consecuentemente, se destaca la importancia de desarrollar alternativas a partir de políticas de cooperación sur-sur orientadas por agendas endógenas. Para ello se presenta como estudio de caso un programa de alcance regional de promoción de redes de investigación motorizado, en el periodo 2013-2015, por el Núcleo de Estudios e Investigaciones en Educación Superior del Sector Educativo del Mercosur.

Palabras clave: Internacionalización Universitaria; Cooperación Sur-Sur; Redes.

\section{University Internationalization and South-South Cooperation Networks}

Abstract: The main problems presented by university internationalization in Latin America, when it is uncritically incorporated, and the potential to dispute its meaning based on the promotion of South-South cooperation policies are analyzed. From a perspective of situated, relational and political analysis, the hegemonic concept of internationalization is discussed, which is reproduced from asymmetric North-South cooperation dynamics and based on research agendas exogenous to the Latin American Region. The importance of developing alternatives based on South-South cooperation policies oriented by endogenous agendas is highlighted. For this, a regional program for the promotion of motorized research networks is presented as a case study, in the period 2013-2015, by the Nucleus of Studies and Research in Higher Education of the Educational Sector of Mercosur.

Keywords: University Internationalization; South-South cooperation; Networks.

\section{Internacionalização Universitária e Redes de Cooperação Sul-Sul}

Resumo: No presente artigo, são analisados os principais problemas apresentados pela internacionalização universitária na América Latina, ao ser incorporada acriticamente, bem como as possibilidades de disputa de seu significado a partir da promoção de políticas de cooperação Sul-Sul. Numa perspectiva de análise situada, relacional e política, discutiremos o conceito hegemônico de internacionalização, que se reproduz a partir de dinâmicas de cooperação norte-sul assimétricas, com base em agendas de pesquisa exógenas à região latino-americana. Destacaremos a importância de desenvolver alternativas baseadas em políticas de cooperação Sul-Sul orientadas por agendas endógenas. Para tanto, apresentamos, como estudo de caso, um programa regional de fomento a redes motorizadas de pesquisa, no período 2013-2015, pelo Núcleo de Estudos e Pesquisas em Educação Superior do Setor Educacional do Mercosul.

Palavras-chave: Internacionalização universitária; Cooperação Sul-Sul; Redes

\section{Ciencia Tecnología y Política \\ Año $4 N^{\circ} 6$ Mayo 2021}

María Soledad Oregioni

Dra. en Ciencias Sociales y Humanas

Centro de Estudios

Interdisciplinarios en

Problemáticas Internacionales y

Locales (UNICEN-CONICET) soregioni08@hotmail.com

Año $4 N^{\circ} 6$ Mayo 2021 e057 Fecha de recibido: 15/02/2021 Fecha de aprobado: 27/04/2021 https://doi.org/10.24215/26183188e057 https://revistas.un|p.edu.ar/CTyP

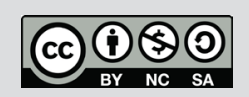

Esta obra está bajo licencia Creative Commons Atribución-NoComercial-Compartirlgual 4.0 Internacional http://creativecommons.org/licenses/bync-sa/4.O/deed.es_AR 
| Ciencia, Tecnología y Política | Año 4 | Nº | e057 | Mayo 2021 | ISSN 2618-3188 | www.revistas.un|p.edu.ar/CTyP |

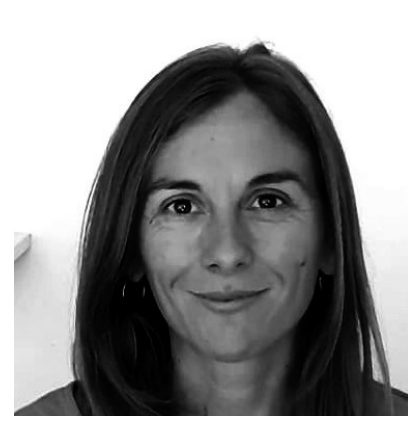

\section{María Soledad Oregioni}

Dra. en Ciencias Sociales y

Humanas

Centro de Estudios Interdis-

ciplinarios en Problemáticas

Internacionales y Locales

(UNICEN-CONICET)

soregioni08@hotmail.com

\section{Internacionalización Universitaria y Redes de Cooperación Sur-Sur}

Resumen: A lo largo del artículo se analizan las principales problemáticas que presenta la internacionalización universitaria en América Latina, cuando es incorporada en forma acrítica, y las potencialidades de disputar su sentido a partir de la promoción de políticas de cooperación sur-sur. Desde una perspectiva de análisis situada, relacional y política, se discute el concepto hegemónico de internacionalización, que se reproduce a partir de dinámicas de cooperación asimétricas norte-sur y en base a agendas de investigación exógenas a la Región Latinoamericana. Consecuentemente, se destaca la importancia de desarrollar alternativas a partir de políticas de cooperación sursur orientadas poragendas endógenas. Para ello se presenta como estudio de caso un programa de alcance regional de promoción de redes de investigación motorizado, en el periodo 2013-2015, por el Núcleo de Estudios e Investigaciones en Educación Superior del Sector Educativo del Mercosur.

\section{Introducción}

La internacionalización universitaria se presenta en la Región Latinoamericana como un proceso multidimensional, complejo y no neutral, donde intervienen dinámicas globales, regionales, nacionales, institucionales y sectoriales. Las mismas se promueven en forma directa a partir de planes de cooperación, e indirecta a través de políticas de evaluación reflejadas, dentro de la universidad, en dinámicas político-institucionales y socio-cognitivas (Oregioni, 2014). En este sentido, en las universidades latinoamericanas se identifican tensiones entre dinámicas de internacionalización hegemónica y no-hegemónicas (Oregioni y Piñero, 2017).

Las dinámicas hegemónicas manifiestan su tendencia mercantil, competitiva y homogeneizante, así como la dependencia académica respecto de los centros mundiales de producción de conocimiento, que históricamente, han incidido a través de legados coloniales, en la generación de ideas (Beigel y Sabea, 2014). La dependencia científica se profundizó, en el contexto de globalización neoliberal, con el surgimiento de mega redes científicas que repercuten en las dinámicas 
de producción de conocimiento, subordinado a modelos de internacionalización estandarizados. Estos toman como referencia los conocimientos desarrollados en el norte global alejándose de la realidad latinoamericana (Kreimer, 2006).

No obstante, las dinámicas de internacionalización no hegemónicas se sostienen a partir de lazos de cooperación sur-sur de carácter endógeno y solidario, entendiendo a la educación superior como derecho y responsabilidad de los Estados, con el objetivo de democratizar la producción y difusión de conocimiento orientado a problemas sociales. De esta forma, las tensiones que emergen, dan lugar a fisuras en el proceso de internacionalización que permiten cuestionar el orden hegemónico y sostener que otra internacionalización es posible. Entendiendo que es necesario desarrollar una conciencia colectiva que permita centrarse en los problemas del Sur como centro de referencia, valorizando sus recursos, buscando complementariedad y beneficios mutuos en la producción y difusión de conocimiento. Consecuentemente, la dimensión política es fundamental en el análisis, no solo entendida como política pública en relación a un proyecto nacional y regional, sino también como disputa de sentido respecto al conocimiento que se genera.

En este artículo se discute el concepto de internacionalización universitaria analizando la política de cooperación en ciencia, tecnología y universidad, en el marco de un proyecto nacional y latinoamericano, que promueva la creación y desarrollo de redes de cooperación sur-sur.

Para ello se utiliza una estrategia metodológica de carácter cualitativo basada en la triangulación de fuentes, centralmente entrevistas a tomadores de decisiones e investigadores, realizadas en el periodo 2016-2020, relevamiento y análisis de documentos institucionales, y análisis de bibliografía especializada.

En primera instancia se presentan las tensiones que emergen en torno al proceso de internacionalización universitaria, contemplando los aportes provenientes del Pensamiento Latinoamericano en Ciencia, Tecnología y Desarrollo (PLACTED); seguidamente se destaca la importancia de promover redes de cooperación sur-sur a partir analizar el programa Núcleo de Estudios e Investigaciones en Educación Superior (NEIES) del Sector Educativo del Mercosur (SEM) como alternativa al modelo de internacionalización hegemónico.

\section{Internacionalización universitaria}

A finales del siglo XX e inicios del XXI comienza a hacerse referencia al proceso de internacionalización de la educación superior, motivado por cambios de orden cuantitativo y cualitativo en la vinculación internacional de las universidades y de sus investigadores. La movilidad y los vínculos internacionales comienzan a presentar aspectos diferentes, respecto de los que han caracterizado a las universidades desde sus orígenes. En este contexto, en América Latina, se contempla la emergencia de programas de cooperación supra-nacionales que promueven proyectos de investigación e intercambio conectados en red. En particular, en el Mercosur, se observa un incremento en la coordinación de regulaciones y políticas de movilidad de carácter regional.

Por otro lado, también se visualiza un incremento en las redes de cooperación como un instrumento para la internacionalización, ahora bien: ¿Cuál es la pertinencia que adquieren las redes respecto a las problemáticas regionales? ¿Cómo inciden las relaciones asimétricas de poder-saber en su dinámica y orientación? En este sentido, se han 
registrado miradas críticas, que identifican problemas derivados de las asimetrías de poder entre los diferentes nodos, diferenciando centros y periferias del conocimiento (Kreimer, 2006; Vessuri, 2013; entre otros).

Siguiendo la perspectiva teórica de Boaventura de Sousa Santos, la universidad en vinculación con otros actores sociales, como el Estado, ocupa un lugar central en la construcción de alternativas contra-hegemónicas a la globalización neoliberal. Estos actores pueden orientar el proceso de internacionalización, mediante políticas de cooperación que permitan la construcción de conocimiento relevante en interacción con otros actores sociales y tipos de saberes (de Sousa Santos, 2008). Sin embargo, este proceso no es lineal. Las tensiones emergen, por ejemplo, a partir de las políticas de evaluación (incidencia indirecta) que promueven lógicas competitivas y homogeneizantes. Un ejemplo de este proceso es la competencia de las universidades por posicionarse en rankings internacionales, retroalimentando un circuito de mercantilización donde el sistema de publicaciones contribuye a orientar la cultura evaluativa en los términos de la "corriente principal" (Beigel, 2019) desde una perspectiva exógena y colonial.

\section{Problematizando la internacionalización hegemónica}

EI PLACTED ofrece elementos para problematizar las desventajas que presenta el proceso de internacionalización hegemónico y pistas para avanzar en nuevos abordajes desde una perspectiva situada, histórica y contextual. Referentes de esta corriente de pensamiento cuestionaron oportunamente la transferencia acrítica y descontextualizada de ideas y formatos institucionales, promoviendo el pensamiento propio (Vaccarezza, 1998).
Varsavsky (1972) sostiene que la ciencia no es neutral, consecuentemente, el desarrollo científico y tecnológico no consiste en "cerrar la brecha" sino en generar un "estilo propio" con contenidos, problemas prioritarios y métodos de investigación particulares. Este "estilo propio" debe ser consistente con un proyecto nacional específico, no copiado de ningún modelo exógeno (Varsavsky, 1972). De este modo la interacción con la ciencia mundial debería darse a través de una percepción crítica, ya que la autonomía científica consiste justamente en tener una independencia de criterio que permita generar conocimiento a partir de las necesidades y valores de nuestras sociedades. Por otro lado, Herrera (1971) plantea como una temática central la cooperación regional para generar capacidad científica autónoma, en base a tomar decisiones de acuerdo a las propias necesidades y objetivos, desde una perspectiva latinoamericana.

Los aportes del PLACTED son recuperados posteriormente por referentes del campo Ciencia, Tecnología y Sociedad (CTS) cuando hacen referencia a las relaciones de poder que median los intercambios científicos en un sistema internacional jerárquico. En particular, se destaca la incidencia de los vínculos internacionales en las dinámicas de producción de conocimiento. Kreimer (2014) diferencia distintos momentos de la internacionalización de la investigación en América Latina y su contribución en la construcción de campos científicos. Señala que la negociación de los problemas cognitivos a trabajar se tornó más dependiente de los centros de producción de conocimiento de los países centrales, generando contratos cerrados, de tipo "tómalo o déjalo", a partir del surgimiento de mega-redes. En este contexto, "Europa y Estados Unidos han ingresado en una competencia global por el desarrollo de capacidades para la innovación e investiga- 
ción científica dentro del contexto de una estrategia competitiva mucho más amplia" (Kreimer, 2014: 153). Esto impacta en la integración subordinada que ha sufrido históricamente la investigación latinoamericana, a partir de: 1) restricción del margen de negociación por parte de los grupos periféricos; 2) proceso de división internacional del trabajo científico que asigna a los investigadores latinoamericanos un lugar fuertemente especializado, con alto contenido técnico, pero con poca incidencia en la definición de los problemas cognitivos a trabajar; 3) reproducción ampliada que permitirá incorporar nuevos métodos y técnicas que luego utilizarán al retomar a sus países. Además, de acuerdo al autor "este nuevo tipo de internacionalización deja poco margen para reformular problemas sociales y locales como problemas del saber" (Kreimer, 2014: 158). Consecuentemente, las asimetrías dificultan la posibilidad de negociar los problemas cognitivos a trabajar y llevan a direccionar las agendas de investigación exógenamente.

Además, con respecto a la movilidad, los datos presentados por la UNESCO (Instituto Internacional de la UNESCO para la Educación Superior en América Latina y el Caribe [IESALC], 2019), sobre los flujos de movilidad académica en América Latina, son reveladores. América Latina y el Caribe son destinos poco elegidos internacionalmente. De los 5 millones de estudiantes a nivel mundial que participan de programas de movilidad, solo el 3,5\% del total escogen como destino algún país de esta región (y de estos el 70\% provienen de América Latina). De los 312 mil estudiantes que migraron de algún país de América Latina y el Caribe para estudiar en otro en 2017, 120 mil (38\%) se quedaron en la propia región mientras que 170 mil (54\%) escogieron como destino Norteamérica o Europa occidental (IESALC2019), contribuyendo a reproducir la circulación de conocimiento orientada desde el norte.

En este sentido, desde la década del sesenta, los estudios sobre dependencia académica, han reunido a importantes investigadores de África, Asia y América Latina que cuestionan el colonialismo y sus efectos (Pinhero y Martin, 2014). De acuerdo con Beigel y Sabea (2014: 15), "el concepto de dependencia académica se refiere a la estructura desigual de producción y difusión del conocimiento construida históricamente en lo que conocemos como sistema científico internacional". Sin embargo, la autora se aleja de una mirada lineal y simplista del fenómeno e identifica el concepto de "heterogeneidad estructural" que refiere a la complejidad de las asimetrías (Beigel, 2013).

En la misma línea, Sousa Santos plantea la incidencia de las relaciones de poder/saber en las dinámicas de producción de conocimiento, afirmando que hasta ahora ese conocimiento científico ha estado concentrado en los países del norte (de Sousa Santos, 2017). Incluso Estados Unidos y Francia libraron disputas por ejercer hegemonía cultural a nivel internacional a partir de programas de asistencia técnica (Bayle, 2015) en el marco de una geopolítica del conocimiento que históricamente se orientó desde el norte, y que ha mediado los vínculos de cooperación intra-regional. A pesar de la cercanía en términos históricos y culturales entre los países latinoamericanos, predominaron los lazos mediados por Estados Unidos y Europa (Marí, et. al; 2001; Kern, 2014). Esto se refleja en estudios bibliométricos que analizan la interacción entre investigadores latinoamericanos (De Filippo et al., 2008; Morales, 2012, entre otros, en Oregioni 2014).

Así, la principal crítica a las redes asimétricas norte-sur consiste en que generalmente llevan a investigar problemas que no responden al contexto donde se desarrolla la investigación, ya que los 
países "periféricos" integran redes muy amplias cuyos programas ya han sido sólidamente estructurados. Esto se puede observar, por ejemplo, a partir de la participación de investigadores latinoamericanos en consorcios promovidos por la Unión Europea (Feld y Kreimer, 2020). Por consiguiente, se cuestiona la presencia de objetivos compartidos y beneficios mutuos sin reconocer las asimetrías de poder que median el proceso de producción de conocimiento.

Consecuentemente, es necesario trabajar en relación a la legitimidad y pertinencia del conocimiento. En este sentido, la cooperación sur-sur permitiría a los países del Sur generar poder de agencia, en un contexto internacional jerárquico, a partir de promover redes de cooperación que orienten la producción de conocimiento hacia agendas de investigación endógenas. Se parte del hecho de que

(...) una agenda constituye un 'conjunto de problemas, demandas, cuestiones y asuntos que los actores sociales han seleccionado y ordenado como objetos de su acción, y como objetos sobre los cuales han decidido que deben actuar o han considerado que tienen que actuar' (Aguilar Villanueva, 1993: 29). En este marco una agenda de investigación refiere a la orientación de la producción de conocimiento científico y tecnológico (Juárez y Castañeda, 2017: 136); y una agenda de investigación endógena orienta la producción de conocimiento científico y tecnológico, en relación a un proyecto nacional y regional (Oregioni, 2018: 30)

El cual debe contemplar, desde una perspectiva dialógica, a los sectores sociales más postergados.

\section{Alternativas al proceso de internacio- nalización hegemónico desde el Sector Educativo del Mercosur}

A partir del Plan Estratégico 2006-2010, elaborado por Sector Educativo del Mercosur (SEM, 2005), se creó el Núcleo de Estudios e Investigaciones en Educación Superior (NEIES), en el contexto de una política que promueve la generación de circuitos latinoamericanos de producción de conocimiento. La propuesta nace en el periodo de regionalismo pos-hegemónico (Tussie y Rigazzi, 2012), donde Argentina orientaba sus vínculos internacionales en relación a un proyecto nacional y regional más amplio. A partir del Consenso de Buenos Aires'슬 en el periodo 2003-2014, el MERCOSUR otorgó más importancia a la dimensión social, política y educativa. De esta forma, las redes de investigación promovidas desde este organismo contribuirían a orientar el proceso de internacionalización universitaria hacia la integración regional y generar conocimiento vinculado a agendas de investigación endógenas a Latinoamérica.

Desde sus orígenes, el NEIES buscó facilitar la construcción de un espacio regional que actúe de puente entre el ámbito de toma de decisiones, la formulación de políticas, y el área de producción de conocimiento. El grupo de trabajo se encuentra integrado por representantes del MERCOSUR, quienes proponen lineamientos estratégicos, acciones y seguimiento. Desde este espacio se impulsaron distintas iniciativas a escala regional: se creó la revista digital "Integración y Conocimiento", se realizaron seminarios, encuentros académicos y convocatorias a Redes, entre otras actividades. De acuerdo a una de las funcionarias entrevistadas:

\footnotetext{
1 En el año 2003, se reunieron los entonces presidentes de Brasil y Argentina en Buenos Aires y proyectaron una agenda política que buscaba intensificar la cooperación regional, conocida como "Consenso de Buenos Aires".
} 
| Ciencia, Tecnología y Política | Año 4 | Nº6 | e057 | Mayo 2021 | ISSN 2618-3188 | www.revistas.unlp.edu.ar/CTyP |

El Núcleo surge como una necesidad de la Comisión Regional Coordinadora de Educación Superior del Mercosur, de tener más insumos para la toma de decisiones políticas. Porque en algún momento nos encontrábamos haciendo el Plan estratégico, como tecnócratas y burócratas de escritorio, armando un plan estratégico sin mucha conexión con la realidad, ahí dijimos: ¿Por qué no hacemos como parte del plan estratégico, que haya un

centro que produzca conocimiento adecuado para esto que estamos haciendo, para lo que necesitamos nosotros en el Mercosur? Así surge el NúCLEO. (Entrevistada, comunicación personal, 2020)

Las convocatorias a redes de investigación respondieron a una agenda concreta y el proceso de selección de los proyectos pasó por dos etapas de evaluación, una etapa nacional y una etapa regional. En la primera convocatoria del año 2013, participaron 46 instituciones de educación superior de Argentina, Brasil, Paraguay, Uruguay y Venezuela, y en la segunda, en el año 2015, concursaron 66 instituciones de educación superior a las que se suman Chile, Colombia y Ecuador. Esto da una idea de la magnitud de su alcance.

En ambos casos se buscó generar sinergias entre las investigaciones y las agendas consideradas prioritarias para la Región.

(...) a través del NEIES se ha ido estableciendo una agenda de temas a nivel regional. Las propias redes han generado oportunidades de interacción y diálogo entre los investigadores de varios países de la región, incluso con investigadores que no integran los proyectos financiados por el NEIES, lo cual va generando visiones propias para la solución de problemas complejos, para la internacionalización de la investigación desde una pers- pectiva regional y para promover una ética del conocimiento científico contrapuesta a las lógicas de mercado. (Astur et al., 2020: p. 10)

Las líneas de investigación que orientan las dos convocatorias fueron pensadas en relación con las prioridades del SEM reflejadas en los Planes de Acción 2006-2010 y 2011-2016 (SEM, 2005; 2010); la Declaración de Belo Horizonte del año 2006; y las declaraciones resultantes de las Conferencias Regionales de Educación Superior (CRES) (IESALC, 2008). Es decir, que surgieron de espacios que propiciaron el debate sobre las universidades en América Latina.

De acuerdo con la directora del Programa de Internacionalización de la Educación Superior y Cooperación Internacional (PIESCI) y coordinadora argentina ante el NEIES, "La declaración de la CRES (2008) sentó las bases de las políticas que se diseñaron desde el PIESCl, entre las que se encuentran las convocatoria a Redes NEIES" (Entrevista a Larrea, comunicación personal, 2020). Además, la entrevistada, hace referencia a que la agenda de trabajo en cooperación internacional se formuló en correlación a los lineamientos de la política exterior, que en ese momento histórico, priorizaba la vinculación con Brasil a nivel bilateral, y con el Mercosur a nivel multilateral.

El rol de Argentina fue fundamental en la conducción y financiamiento del programa, en tanto, funcionarios de los distintos países acompañaron la iniciativa a partir de formar parte de los grupos de trabajo. De acuerdo al informe de evaluación institucional del NEIES (Astur et al., 2020), el formato y orientación de las redes se encuentra en sintonía con las políticas nacionales del periodo. Destaca además la importancia de la cooperación entre los grupos de investigación de la región, y resalta que el $94 \%$ de los investigadores que participaron de las convocatorias pertenecen a universida- 
des públicas.

Al indagar en la dinámica de producción de conocimiento que se genera desde las redes, los investigadores participantes coinciden en señalar que les permitió incrementar los vínculos intra-regionales desde una perspectiva integral, dando lugar a publicaciones conjuntas; actividades de extensión, como: cursos, talleres, seminarios y consolidar los vínculos académicos con las universidades del MERCOSUR (Entrevistas a coordinadores de Redes NEIES, Comunicación personal, 2020). Además, el informe de evaluación del programa (Astur, et. al., 2020) da cuenta del incremento de la interacción a nivel regional, esto es reafirmado en las entrevistas a directivos, cuando señalan que: "Si se toma como punto de partida el estado de arte relevado por el NEIES en el año 2009, el crecimiento de iniciativas de investigación entre grupos de universidades de la región ha sido notorio, y las redes han estado involucradas de modo más o menos directo". (Entrevista a Larrea, comunicación personal, 2020)

El programa logró generar redes, desde una perspectiva interdisciplinar, dado que se abrieron diferentes líneas de investigación en relación a las agendas propuestas (Coordinadores de Redes NEIES, comunicación personal, 2016). También lograron hacer aportes en la generación de capacidades.

El perfil de los integrantes de la red es de docencia, investigación y gestión, en el caso de Argentina, Brasil y Uruguay, y en el caso de Paraguay, de docencia y gestión (...) es necesario fortalecer la investigación, de hecho, han sido los que tuvieron más dificultades en la recolección de datos (...) pero se logró generar un instrumento que aplicaron a las distintas universidades, y las universidades respondieron. Creo que fue un aporte impor- tantísimo. (Entrevista a participante de Red NEIES, comunicación personal, 2017).

La convocatoria planteó como objetivo explícito: incrementar los vínculos entre investigadores latinoamericanos para generar conocimiento pertinente a nuestras realidades. En este sentido, se priorizó el criterio de legitimidad por sobre los de eficiencia y eficacia (Oregioni y Piñero, 2009). Sin embargo, de acuerdo a la coordinación del NEIES, la relevancia del programa no solo se constata en la coherencia con el enfoque y objetivos planteados en el SEM, sino también en las sinergias generadas con otros espacios regionales como las Conferencias Regionales de Educación Superior de la UNESCO para América Latina; la Asociación de Universidades del Grupo Montevideo (AUGM); y los grupos de investigación que trabajan en la temática, entre otros.

De esta forma, las redes creadas a partir de la convocatoria lograron adquirir presencia en relación a las agendas endógenas de la región. Además, permitieron generar un tejido relacional de carácter regional vinculado a las principales problemáticas que afectan a las universidades latinoamericanas en el siglo XXI, desde una perspectiva de cooperación solidaria, que permite dar cuenta de dinámicas de internacionalización alternativas a la globalización neoliberal y neocolonial.

Esto condujo a fortalecer los grupos de investigación y generar nuevos espacios de producción de conocimiento colaborativo, pero no ha logrado repercutir sobre la toma de decisiones a nivel nacional y regional en el periodo 2016-2019. Si bien el programa del NEIES se creó en el contexto del regionalismo pos-hegemónico, el giro a la derecha en la región, y en Argentina en particular, afectó su continuidad político-institucional, dado que no se renovó la convocatoria. A esto se suma la falta de apoyo, en esta etapa, de Brasil a partir del 
Programa "Ciencias sin Fronteras" que contribuyó a erosionar el incipiente espacio de cooperación regional.

No obstante, a nivel socio-cognitivo, los resultados de las dos convocatorias muestran que se ha dinamizado la circulación de conocimiento entre los grupos de investigación, se ha incrementado la masa crítica y la producción de conocimiento orientada a una agenda construida en forma conjunta, y se ha ampliado la divulgación intra-regional. Es decir que, tuvo buena recepción por parte de la comunidad académica, que excedió el campo de estudios sobre educación superior, incorporando a docentes, investigadores, estudiantes, extensionistas y gestores interesados en la universidad.

\section{Conclusión}

El análisis realizado pone en evidencia la importancia de disputar el sentido del proceso de internacionalización, puntualizando su carácter no neutral en un contexto internacional asimétrico, que condiciona en gran medida las agendas de investigación y, consecuentemente, las características del conocimiento que se genera. De ahí la necesidad de poder contar con una política de cooperación regional que permita orientar la internacionalización universitaria en relación a un proyecto nacional y regional más amplio, que estimule a pensar la universidad desde la Región, y la Región desde la universidad en función del bienestar de nuestros pueblos.

El caso de estudio, permitió identificar la importancia de trabajar en relación a una agenda endógena para el Sector Educativo del Mercosur, mostrando que en el contexto de regionalismo pos-hegemónico se generaron dinámicas de circulación de conocimiento entre universidades del Mercosur y aprendizajes políticos-institucionales.
Estas dinámicas se lograron sostener hasta el año 2015, cuando el giro a la derecha en la Región y el retorno del neoliberalismo en la Argentina, impidieron profundizar el proyecto no abriendo nuevas convocatorias y dándole prioridad al vínculo neocolonial con los países del norte, que forma parte de la lógica de internacionalización hegemónica.

Sin embargo, la experiencia da cuenta de que cuando existe compromiso político, se pueden explorar las fisuras del proceso de internacionalización hegemónico mediante la promoción de dinámicas de cooperación sur-sur. En el Sector Educativo del Mercosur esto posibilitó la generación de aprendizajes y de un tejido socio-cognitivo y político-institucional que debe ser retomado por los sectores progresistas de la región, a partir de orientar la internacionalización universitaria hacia la integración Latinoamericana.

\section{Bibliografía}

Astur, A.; Gandola, M.; Isasmendi, G. y Pazos, N. (2020). Informe de Evaluación sobre el programa redes de investigación. Núcleo de Estudios e Investigaciones en Educación Superior del MERCOSUR. Secretaría de Políticas Universitarias, Ministerio de Educación. Argentina.

Bayle, P. (2015). Conectando sures. La construcción de redes académicas entre América Latina y África. Iconos. Revista de Ciencias Sociales. Núm. 53, 153-17. https://doi.org/10.17141/ iconos.53.2015.1445

Beigel, F. (2013). Centros y periferias en la circulación internacional del conocimiento. Nueva Sociedad (245), 110-123.

Beigel, F. (2019). Indicadores de Circulación, una perspectiva multiescalar para medir la producción científico-tecnológica latinoamericana. Revista 
Ciencia, Tecnología y Política. Año 2, № 3. https:// doi.org/10.24215/26183188e028

Beigel, F. y Sabea, H. (2014). Dependencia Académica y Profesionalización en el Sur. Perspectivas desde la Periferia. Universidad Nacional de Cuyo.

de Sousa Santos, B. (2008). El rol de la universidad en la construcción de una globalización alternativa. La Educación Superior en el mundo. Universidad Politécnica de Catalunya.

de Sousa Santos, B. (2017). Justicia entre saberes. Epistemologías del sur contra el Epistemicidio. Ed. Morata.

Feld, A. y Kreimer, P. (2020). Científicos latinoamericanos en proyectos europeos: Asimetrías en la Cooperación Científica Internacional. Revista Ciencia, Tecnología y Política. Año 3 n4. https:// doi.org/10.24215/26183188e035

Herrera, A. (1971). Ciencia y política en América Latina. Siglo Veintiuno.

Instituto Internacional de la UNESCO para la Educación Superior en América Latina y el Caribe (2008). Declaración y plan de acción de la Conferencia Regional de Educación Superior en América Latina y el Caribe. https://unesdoc.unesco.org/ ark:/48223/pf0000181453.locale=es

Instituto Internacional de la UNESCO para la Educación Superior en América Latina y el Caribe (2019). La movilidad en la Educación Superior en América Latina y Caribe: retos y oportunidades de un convenio para el reconocimiento de estudios, títulos y personas. https://unesdoc.unesco.org/ ark:/48223/pf0000372629.locale=es

Kern, A. (23-25 de julio de 2014). La agenda científica y tecnológica en los regionalismos de América Latina. Conferencia Internacional Conjunta FLACSO-ISA, 1-22.

Kreimer, P. (2006). ¿Dependientes o integrados?
La ciencia latinoamericana y la división internacional del trabajo. Nómadas. № 24, 199-212.

Kreimer, P. (2014). ¿Ciudadanos del Mundo o productores de conocimiento útil? Esa es la cuestión, en Sabea, H. y Beigel, F. (coord.). Dependencia Académica y Profesionalización en el Sur. Perspectivas desde la Periferia. Universidad Nacional de Cuyo.

Marí, M.; Estébanez, M.; Suarez, D. (2001). La Cooperación En Ciencia y Tecnología de Argentina con los Países del Mercosur. Revista Redes, VIII, (17), 59-82.

Oregioni, M. (2014). Dinámica de la Internacionalización de la Investigación en la Universidad Nacional de La Plata (2005-2012) [Tesis de Doctorado, Universidad Nacional de Quilmes]. https://ridaa. unq.edu.ar/bitstream/handle/20.500.11807/120/ OREGIONI.pdf?sequence $=1$ \&isAllowed $=y$

Oregioni, M. (2018). Redes de producción de conocimiento y dinámicas de cooperación sur-sur ¿Una alternativa a la internacionalización universitaria hegemónica? en Oregioni, M., y Taborga, A. (comp.), Dinámicas de Internacionalización Universitaria en América Latina (pp. 19-40). Grafikart.

Oregioni, M. y Piñero, F. (2009). Eficacia y legitimidad en las políticas de cooperación en ciencia y tecnología en el MERCOSUR. Un aporte para su interpretación. Revista APORTES, 27-42.

Oregioni, M. y Piñero, F. (2017). Las redes como estrategia de internacionalización universitaria en el Mercosur. El caso de la RIESAL (2013-2017). Revista Integración y Conocimiento, 114-133.

Pinheiro, C. y Martín, E. (2014). SEPHIS y la crítica a la Dependencia Académica en el Mundo Actual en Beigel, F. y Sabea, H. (coord.), Dependencia Académica y Profesionalización en el Sur (pp 1114). EDIUNC; SEPHIS. 
Sector Educativo del MERCOSUR (2005). Plan Estratégico del Sector Educativo del Mercosur 2006-2010. http://www.bnm.me.gov.ar/giga1/documentos/EL006482.pdf

Sector Educativo del MERCOSUR (2010). Plan Estratégico del Sector Educativo del Mercosur 20112016. http://www.bnm.me.gov.ar/giga1/documentos/EL006482.pdf

Tussie, D. y Riggirozzi P. (2012). The Rise of Posthegemonic Regionalism. The Case of Latin American. Springer.

Vaccarezza, L. (1998). Ciencia, Tecnología y Sociedad: el estado de la cuestión en América Latina. Revista Iberoamericana de Educación, Nº 18, 13-40.

Varsavsky, O. (1972). Hacia una politica científica nacional. Periferia.

Vessuri, H. (2013). El nuevo mantra de la diplomacia científica internacional: ¿Co-diseño de conocimiento? ¿Investigación integrativa? Universitas 76, 25-50. 